\title{
Philosophiques
}

Bonnet, Nicole, Immanence et transcendance chez Teilhard de Chardin. Préface d'André A. Devaux, Montréal/Paris, Bellarmin/Cerf, 1987, 324 p. (Coll. Recherches, Nouvelle Série : 11).

\section{Simone Plourde}

Volume 15, numéro 2, automne 1988

URI : https://id.erudit.org/iderudit/027060ar

DOI : https://doi.org/10.7202/027060ar

Aller au sommaire du numéro

Éditeur(s)

Société de philosophie du Québec

ISSN

0316-2923 (imprimé)

1492-1391 (numérique)

Découvrir la revue

Citer ce compte rendu

Plourde, S. (1988). Compte rendu de [Bonnet, Nicole, Immanence et transcendance chez Teilhard de Chardin. Préface d'André A. Devaux,

Montréal/Paris, Bellarmin/Cerf, 1987, 324 p. (Coll. Recherches, Nouvelle Série :

11).] Philosophiques, 15(2), 487-489. https://doi.org/10.7202/027060ar d'utilisation que vous pouvez consulter en ligne.

https://apropos.erudit.org/fr/usagers/politique-dutilisation/ 
BONNET, NICOLE, Immanence et transcendance chez Teilhard de Chardin. Préface d'André A. Devaux, Montréal/Paris, Bellarmin/Cerf, 1987, 324 p. (Coll. Recherches, Nouvelle Série: 11).

\section{par Simone Plourde}

Entre les années 1950 et 1970, la pensée de Teilhard de Chardin a suscité une abondante littérature. Les études qu'on lui a alors consacrées reflétaient les controverses soulevées par l'évolutionnisme teilhardien et certaines critiques déniaient à l'ensemble de l'œuvre la valeur scientifique revendiquée par son auteur.

Avions-nous besoin aujourd'hui d'une nouvelle étude sur l'œuvre de Teilhard? Le teilhardisme serait-il l'une de ces grandes pensées qui surgissent trop tôt et qui bousculent à ce point notre vision des choses qu'il faut les laisser en veilleuse un moment avant d'en saisir la pertinence? Nicole Bonnet a sans doute estimé que le temps était venu de faire le point et de présenter au public une étude où apparaît la taille scientifique, philosophique et religieuse de Teilhard. Pour entreprendre l'analyse qu'elle nous offre dans son livre, il lui a certes fallu percevoir dans la pensée teilhardienne une valeur et une actualité qui justifient sa patiente et courageuse tentative.

C'est en philosophe que l'A. aborde l'œuvre de Teilhard. Elle a discerné dans celle-ci un fil conducteur qui lui a permis de mettre en relief la progression d'une pensée aux multiples facettes : la relation étroite entre les concepts d'immanence et de transcendance. Nicole Bonnet s'applique donc à montrer comment se joue la dialectique de l'immanence et de la transcendance aux niveaux suivants: la vie, l'homme, la société, l'action. Cette voie la conduit à la clef maîtresse de l'œuvre de Teilhard, jusqu'à l'absolu que postule l'évolution, c'est-à-dire le " point Oméga ». Immanence donc et transcendance du point Oméga identifié au Christ, immanence et transcendance de l'Esprit qui justifient toute la conception teilhardienne des œuvres de l'effort et du progrès.

Ce qui fait l'originalité de la pensée de Teilhard et apparaît comme central chez lui est très bien mis en relief, à savoir le «souci de découvrir le sens de la vie et de l'histoire, de déceler dans la réalité elle-même le sens de l'évolution, d'élucider son intentionnalité immanente dans l'ordre du phénomène par la seule méthode scientifique» (p. 47).

À quelques reprises, Nicole Bonnet souligne de façon pertinente l'originalité de la méthode teilhardienne qui, écrit-elle, " pénètre le réel progressivement, par conjonctions d'axes de pénétration scientifique, philosophique, religieuse, par une "série de réflexions successives", par un mouvement alternatif de prospection et de rétrospection, par retournement, si bien que tout s'éclaire par le final qui est essentiellement le "finalisant". La dialectique teilhardienne est un retournement où l'immanence et la transcendance sont mise en relation réciproque » (p. 187). Chez Teilhard, la méthode du retournement, c'est explique-t-elle, un changement de pôle, une excentration qui 
fait passer l'auteur d'un centre donné à un centre supérieur : méthode qui permet à Teilhard d'opérer le passage de la phénoménologie à l'ontologie.

Il est impossible de rendre compte ici en détail de l'étude de Nicole Bonnet qui suit pas à pas le cheminement de la pensée teilhardienne, de la loi de complexité-conscience jusqu'au Dieu de l'En-Haut et de l'En-Avant. Mentionnons que l'A. n'élude pas les objections apparentes ou réelles que soulève la pensée de Teilhard, qu'elle les discute, qu'elle pointe bien les convergences et les divergences entre, d'une part, Teilhard, et, d'autre part, Bergson, Mounier, Blondel, Ernst Bloch, le marxisme, Monod, etc. ; qu'elle n'ignore pas les accusations de syncrétisme, de panthéisme, d'optimisme, erc. portées contre le teilhardisme, mais tente de dénouer les malentendus et les impasses.

C'est à l'immanence et à la transcendance du Christ qu'est consacré le plus long chapitre du livre, thème où se rejoignent le plan phénoménologique et le plan religieux, point brûlant autour duquel se sont souvent noyautées plusieurs objections à la « sainte Évolution ». C'est à l'intérieur de ce chapitre qu'est très bien explicitée la catégorie de transformation créatrice, fruit d'une réflexion de Teilhard qui date de 1919, au moyen de laquelle ce penseur, à la fois homme de science réputé et philosophe, opère le dépassement dialectique de la transformation et de la création. Disons en bref ce que développe longuement l'A.: pour Teilhard, créer, c'est unir et non pas formellement produire. L'Un ne lui apparaît qu'à la suite du Multiple, dominant celui-ci, puisque son action formelle est d'unir. Nicole Bonnet dégage bien les deux postulats qui soutiennent la théorie de l'union créatrice - théorie induite du réel, option et principe de base d'une construction qui en est réduite, théorie qui s'attache à élucider la genèse de l'Esprit au cours de l'unification du Multiple, - à savoir : 1) l'être est bon; 2) la vie réussit.

Le livre de Nicole Bonnet ouvre-t-il de nouvelles perspectives sur la pensée de Teilhard de Chardin? Il nous faudrait avoir lu l'abondante littérature que cette dernière a suscitée pour répondre adéquatement à cette question. Nous croyons que le fil directeur pressenti et bien exploité par l'A. laisse voir sous un angle nouveau l'unité profonde de l'cuvre teilhardienne. Il nous autorise à donner raison à l'A., lorsqu'elle écrit : "Passionné d'unité, Teilhard a réalisé l'union entre l'immanent et le transcendant, l'union de la science et de la foi, au niveau de son expérience vécue, de sa phénoménologie, de sa philosophie, de l'action " (p. 313).

Nicole Bonnet fait preuve d'une connaissance approfondie de l'œuvre de Teilhard de Chardin dont elle s'applique à exposer et à suivre les nervures principales sans se perdre dans les détails, passionnants certes, mais qui obnubilent l'architecture de la pensée. Son étude est une invitation à dépasser la lecture du Phénomène Humain, d'allure plus scientifique, à entrer dans l'envergure d'une pensée qu'éclairent deux foyers de lumière formellement distincts, mais qui conjuguent ici leurs faisceaux lumineux : la raison et la foi. Il faut sans doute être croyant pour suivre Teilhard jusqu'au bout et se livrer comme lui sans réticence au souffle d'espérance qui portait son esprit et 
animait tout son être. Il faut au moins être sensible et ouvert à la dimension de la transcendance pour résister à la tentation d'amputer cette cuvre des éléments essentiels sans lesquels elle perd sa signification.

Sous la plume de Nicole Bonnet émergent principalement les dimensions métaphysique et morale de l'œuvre de Teilhard. Tel que présenté par l'A., le teilhardisme nous est rendu accessible, simplifié, mais sans réduction indue. Bien loin d'être un simple livre de vulgarisation, l'ouvrage de Nicole Bonnet peut être considéré comme une honnête et excellente introduction à l'ensemble de la pensée de Teilhard de Chardin, dont il met bien en relief tant l'unité structurelle profonde que l'envergure d'une vision qui englobe passé et avenir.

Université du Québec à Rimouski 